\title{
An acoustic communication based AQUA-GLOMO simulator for underwater networks
}

Sanjay K Dhurandher ${ }^{1}$, Mohammad S Obaidat ${ }^{2^{*}}$ and Megha Gupta ${ }^{3}$

\author{
* Correspondence: \\ obaidat@monmouth.edu \\ ${ }^{2}$ Department of Computer Science \\ \& Software Engineering, \\ Monmouth University, NJ, USA \\ Full list of author information is \\ available at the end of the article
}

\begin{abstract}
In an underwater environment there can be various applications relating to the naval monitoring of an area and information gathering through the sensor nodes or underwater vehicles. The information gathered/monitored/detected in this manner can be sent to a group of sensors, or any sensor or vehicle may initiate a query asking for the information of its interest from the subset of the sensors. This information transfer or query dissemination can involve either broadcasting, multicasting or geocasting technique, depending on whether the query is sent to all the nodes, or a subset of nodes based on their locations. After developing any technique, it is required to validate it for its purpose. In underwater scenarios, testing any technique is not only costly but difficult too in terms of full deployment. Hence the next option is to test any modeled technique on a Simulator. A number of simulation tools have already been developed for the wired/wireless radio networks. However, these tools cannot be used for underwater environment with acoustic communication medium, as there is wide range of difference between the radio and the acoustic communications. Therefore, the acoustic communication based AQUAGLOMO simulator has been implemented with GLOMOSIM as the base simulator. At the network layer, the RMTG model has also been included in this simulator that is an underwater sensor network geocasting model. The results obtained on this simulator have shown an evolving efficient simulator for underwater acoustic communications.
\end{abstract}

Keywords: Glomosim, Acoustic Model, Underwater Sensor Networks, Modeling and Simulation, Performance Evaluation

\section{Introduction}

A Model is a simplified representation of an actual system. Modeling is a way to perform the system testing before its actual implementation. Simulation is the process of writing a computer program that implements the model. In simulation analysis, we can conduct simulation runs, often called simulation experiments, in order to understand the behavior of the system and predict its performance at various points of time. In the literature, modeling and simulation are used together due to their tight relations.

A Simulation Tool (Simulator) can create a virtual testing environment which is close to the real scenario. The testing results on the simulator provide the approximate view of the real environment performance.

In recent years, under water sensor networks have been widely used in underwater environment for a wide range of applications, such as pollution monitoring, health

\section{Springer}

(C) 2012 Dhurandher et al; licensee Springer. This is an Open Access article distributed under the terms of the Creative Commons Attribution License (http://creativecommons.org/licenses/by/2.0), which permits unrestricted use, distribution, and reproduction in any medium, provided the original work is properly cited. 
monitoring of marine creatures, sensing naval activities and information gathering. An underwater sensor network (UWSN) [1] consists of a number of sensor nodes which can perform various functions such as data sensing, data processing and communication with the other nodes.

UWSNs are different from ground-based sensor networks in terms of the communication methods and the mobility of the nodes. For communication, UWSNs use acoustic signals instead of radio signals. Acoustic signals are used due to their lower attenuation in underwater environment. The node movements under the water are random due to the unpredictable water current.

A number of researchers are working in the area of underwater networks and they require tools to test their proposed models. Tools are available that allow the formation of acoustic networks beneath the big water bodies, but their deployment is very expensive, difficult and not practical in terms of system testing before system's full implementation.

Moreover a number of challenges lie ahead for the actual working of such real scenarios [2-4]. Hence researchers require simulation tools with acoustic models for testing the working of their proposed models. For underwater environment, the main termination point faced by the researchers is the unavailability of the simulation tools, as there are limited open source simulation kits available for acoustic communication.

Whenever any research in underwater environment is carried out, there is a need to have acoustic communication channel in the physical layer. Hence in this paper, we present a simulation environment for underwater acoustic networks. We take the easily available open source simulation tool GLOMOSIM [5] as our base tool and implement the acoustic model within it for UWSN. We named it as AQUA-GLOMO; GLOMOSIM simulator with ACOUSTIC MODEL at the physical layer and RMTG [1] at the network layer as shown in Figure 1.

The rest of the paper is structured as follows. The next section presents the ACOUSTIC model for UWSNs. In Section III we describe the AQUA-GLOMO simulator. In Section IV we evaluate this simulator. Section V contains the conclusion and future work.

\section{Acoustic model}

The ACOUSTIC model refers to the communication with the sound waves. In underwater environment, communicating medium can be either radio, optical or sound (acoustic) waves. But the non-acoustic waves are electromagnetic waves which suffer from high propagation losses as well as scattering problems. These non-acoustic waves do not travel long distances in underwater environment. Radio waves require high transmission power as well as long antennas to communicate and Optical waves suffer from high signal attenuation so it can travel short ranges only. Hence sound is the best communicating medium for underwater networks. Till now and in near future also, the acoustic waves can be seen as the best communication medium for wireless networks in UWSNs.

The acoustic waves also have some limitations, which need to be considered for reliable data sharing in UWSNs [6]. Major designing challenges with acoustic model are long propagation delay and low available bandwidth. Hence large bulk data transmission is impractical in UWSNs. 


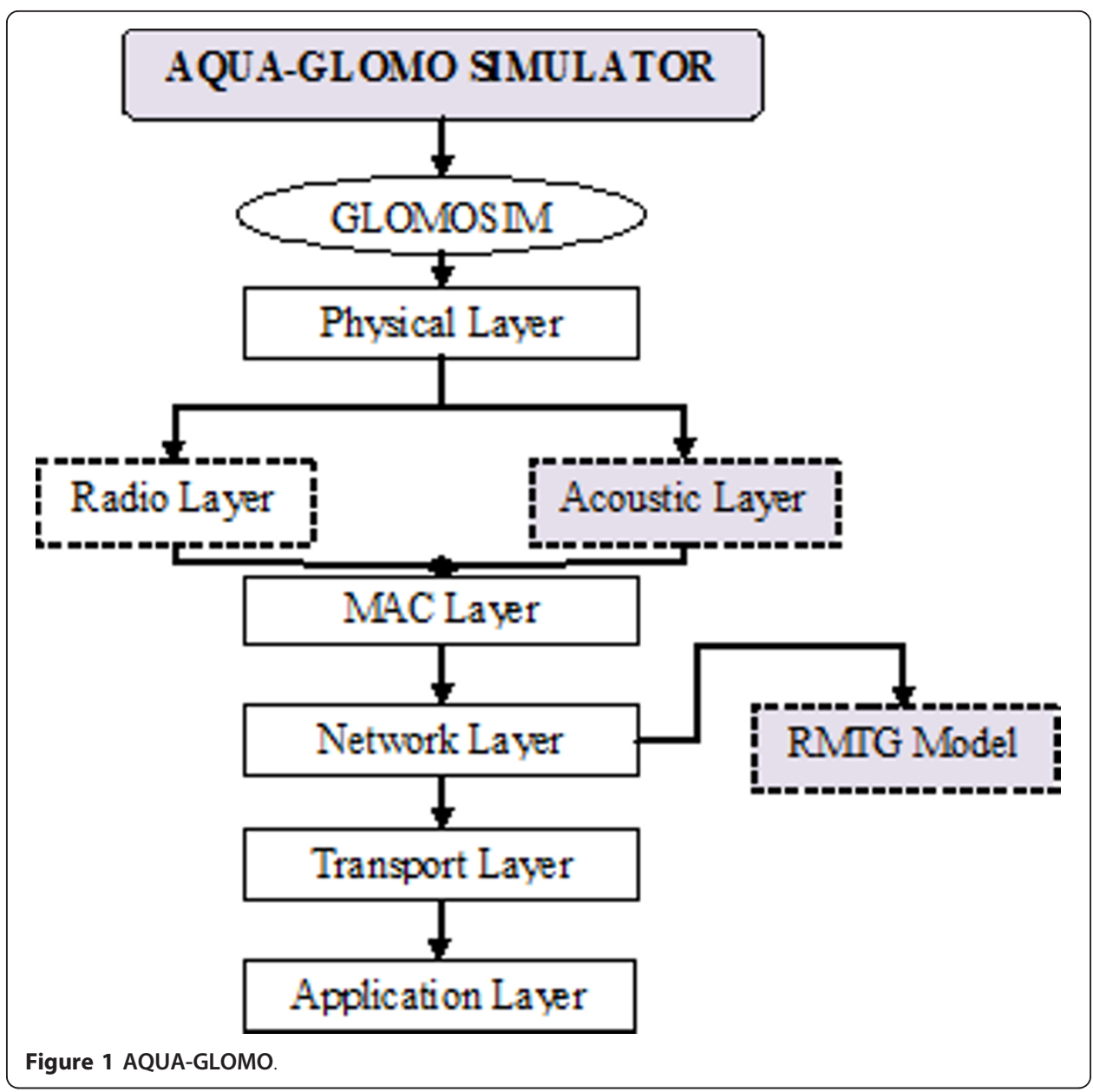

The Acoustic communication can work in 3 frequency bands [7]:

- Very-High frequency band (>50 kHz) - Have short range for communication.

- Moderate frequency band (between $20 \mathrm{kHz}$ to $50 \mathrm{kHz}$ ) - Have medium range of the order of $1 \mathrm{Km}$.

- Low frequency band $(<10 \mathrm{kHz})$ - Have long range for communication of the order of $10 \mathrm{Km}$.

An underwater sensor network scenario with acoustic communication is shown in Figure 2. In Figure 2, the sensor nodes/vehicles are present beneath the water level as well as floating on the surface of the water. Under water, nodes are communicating via the acoustic communication and above the surface of water, the nodes are communicating via the radio signals. There can be a master data collector center or an analysis center which can collect the information from these nodes for various purposes. As shown in Figure 2, the Surface Base Station is serving as the Master Data Collector that is gathering information through various means (radio/acoustic) from data sensed by different types of nodes present in this scenario. 


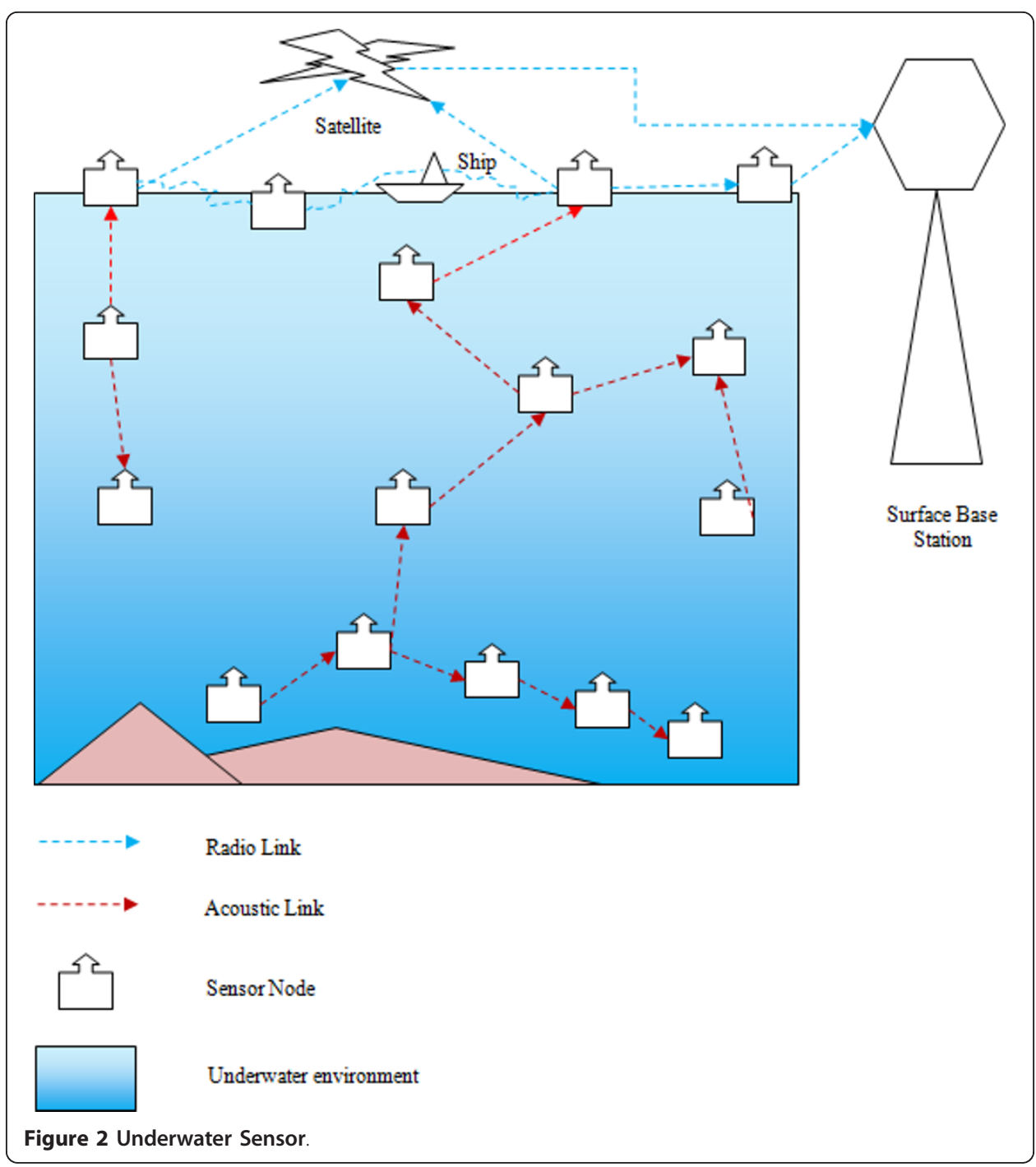

In underwater acoustic model, the wave's attenuation is dependent on two major factors that are:

1. Range of the transmission between the transmitter and receiver.

2. Wave Frequency.

Underwater attenuation $A(l, f)[8]$ can be expressed as:

$$
A(l, f)=\left(\frac{l}{l_{\text {ref }}}\right)^{a l p h a} . a(f)^{l}
$$

\section{Where:}

$L$ : Distance between transmitter and receiver,

$l_{\text {ref }}$ Reference distance (typically $1 \mathrm{~m}$ ),

Alpha: Used to model the geometry of propagation and it is the counterpart of the path loss coefficient in terrestrial radio (its practical value is 1.5),

$a(f)$ : Absorption Loss, which depends on frequency $f$. 
The $a(f)$ value can be calculated using Thorp's formula [8,9] as mentioned below:

$$
10 \log a(f)=\frac{0.11 f^{2}}{1+f^{2}}+\frac{44 f^{2}}{4100+f}+2.75\left(10^{-4} \cdot f^{2}\right)+0.003
$$

Where,

$f=$ Frequency in $\mathrm{kHz}$,

The $a(f)$ value calculated using equation(2) is in $\mathrm{dB} / \mathrm{km}$.

If a tone of frequency $f$ and power $P(t)$ is transmitted over a distance $l$, the received signal power as in [10] will be given by:

$$
P(r)=\frac{P(t)}{A(l, f)}
$$

For underwater acoustic communication, the sound propagation speed $c$ in $\mathrm{m} / \mathrm{s}$ is also dependent on a number of factors (the water depth, its temperature and salinity) [11]. In this paper, we assume $c=1500 \mathrm{~m} / \mathrm{s}$, which is a commonly considered average value. It is worth mentioning here that $c$ has weak dependence on these factors.

\section{Aqua-Glomo Simulator}

\section{A. Motivation}

Simulation analysis is becoming an integral part of various evolving research areas. It helps to better understand the theoretically proposed models and evaluate designs before investing time and money in building them. It also helps to assess various operation scenarios of any systems in order to select the scenario that meets the requirements. In real scenarios, modeling, coding and testing of an actual system is a mountaineer task which requires a mammoth of money, and time. Various simulation tools are easily available that have been designed to aid in the testing of the wired/ wireless radio networks. However, these tools are not suitable for underwater networks that require acoustic communication, as there is a wide range of difference between the radio and the acoustic communications.

The ongoing research efforts in the field of UWSNs require an efficient and easily available simulation tool for designing and analyzing these works. This has been the major motivation factor towards the development of this simulator.

\section{B. Glomosim}

Global Mobile Information System Simulator (GloMoSim) [1,12] provides the simulation environment for large wireless/wired communication networks. It is good for simulating mobile networks. It works with C-based parallel discrete-event simulation language PARSEC (Parallel Simulation Environment for Complex Systems). It can work efficiently in a parallel environment; this property distinguishes it from most other sensor network simulators. It is an open-source and an extensible tool as all other protocols is implemented as modules in its library. Its main limitation is that it only presently supports protocols for a purely radio wireless network; not acoustic.

\section{RMTG}

Routing and Multicast Tree based Geocasting (RMTG) [2] is a geocast technique, designed for underwater sensor networks. The RMTG technique uses greedy 
forwarding and previous hop handshaking to route the packets towards the geocast region and further disseminates the data within the geocast region by creating a multicast shortest path tree. RMTG does not use the flooding technique to deliver the packets inside the geocast region that is used in most of the geocasting techniques. Most of the work on geocasting has been done for mobile ad-hoc networks and vehicular adhoc networks; RMTG is a novel simulated model for underwater environment.

\section{AQUA-GLOMO}

AQUA-GLOMO simulation tool is developed to provide the underwater acoustic communication with the GLOMOSIM simulator. Main characteristics of this tool are tabulated in Table 1. In this tool the coding is done using C-based language, called as PARSEC (Parallel Simulation Environment for Complex Systems). At the Physical Layer, the acoustic communication is implemented. Acoustic signals attenuation is modeled as mention in equation 1 , which is dependent on the distance and the frequency. The acoustic signal speed is taken as $1500 \mathrm{~m} / \mathrm{s}$, which is five times smaller than the speed of light. Absorption Loss occurs when traveling acoustic wave energy is converted to some other form (may be heat) and absorbed by the carrier medium. Using the Thorp's formula, the absorption loss is calculated as stated in equation 2 .

For underwater acoustic communication, the main focusing area is communication and the networking of the nodes. Proper communication can be achieved by implementation of the acoustic model at the physical layer. Efficient networking can be achieved by having a good routing protocol at the network layer. In AQUA-GLOMO at the network layer, we have used our previous work, RMTG model that provides geocasting and routing as the sub-part of this model.

\section{Simulation Analysis}

In this section, the simulation of the AQUA-GLOMO simulator is described. In the simulation runs, the nodes are assumed to be uniformly deployed with Constant Bit Rate (CBR) as traffic generator. The performance metrics considered in our work are the packet delivery ratio and the delay involved in data transfer.

The Packet Delivery Ratio is taken as the ratio of total number of packets that should be in geocast region within the simulated time and the number of packets that reach in the geocast region within the same time period.

The Average End to End Delay is the time taken to reach the data from the root node to the last node of the multicast tree in the geocast region.

Routing delay is the time taken to reach the data from the source node to the root node that is to reach till the geocast region.

Table 1 AQUA-GLOMO Simulator

\begin{tabular}{cl}
\hline Base Simulator & $\cdot$ Glomosim \\
\hline Implementation Language & $\cdot$ C/PARSEC \\
\hline Physical Layer Model & $\cdot$ Signal attenuation model as mentioned in equation (1). \\
& $\cdot$ Absorption Loss is calculated using Thorp's approximation as in equation (2). \\
\hline Network Protocol & $\cdot$ PMTGagation speed is speed of sound $=1500 \mathrm{~m} / \mathrm{s}$ \\
\hline Mobility Model & $\cdot$ Random-Waypoint \\
\hline
\end{tabular}


Table 2 AQUA-GLOMO simulation parameters and their values

\begin{tabular}{cc}
\hline Parameters & Value \\
\hline Data Frame Payload Size & 50 bytes \\
\hline Propagation Speed & $1500 \mathrm{~meter} / \mathrm{sec}$ \\
\hline Data Rate & $10000 \mathrm{bits} / \mathrm{sec}$ \\
\hline Node Frequency & $25 \mathrm{KHz}$ \\
\hline Transmission Power & $1000 \mathrm{~mW}$ \\
\hline Receiving Threshold Power & $20 \mathrm{~mW}$ \\
\hline Range & $750 \mathrm{~m}$ \\
\hline
\end{tabular}

The Simulation parameters used in our work are tabulated in Table 2. The simulations are performed for a simulation time of 92 seconds. The terrain considered is of $2500 \times 2500$ square meters with $1300 \times 1300$ square meters as the geocast region.

For better simulation performance, the following parameters were varied to obtain the results.

1. Nodes without mobility that is the static network condition versus mobile network conditions is evaluated (refer Figure 3).

2 . Nodes mobility - by varying the speed of the moving nodes with values $1 \mathrm{~m} / \mathrm{s}, 3$ $\mathrm{m} / \mathrm{s}$ and $5 \mathrm{~m} / \mathrm{s}$, (refer Figure 4, 5 and 6).

3. Terrain area - by varying the terrain region dimensions with values $2500 \times 2500$, $3000 \times 3000,3500 \times 3500$ square meters, (refer Figure 7, 8 and 9).

4. Geocast region area - by varying the geocast region dimensions with values 1100 $\times 1100,1300 \times 1300,1500 \times 1500$ square meters, (refer Figure 10, 11 and 12)

5. Number of nodes in the terrain area and the geocast region were varied with the change in the settings of the terrain region and the geocast region dimensions, (refer figure from 4 to 12 ).

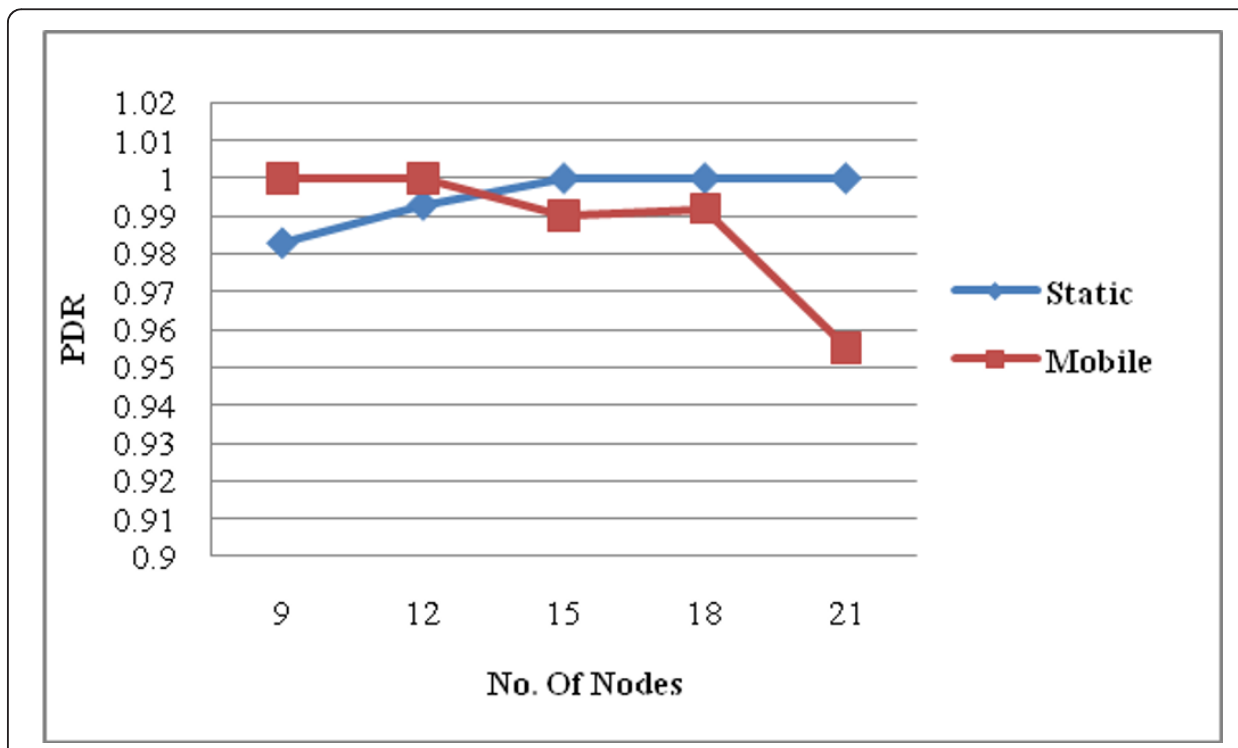

Figure 3 Packet Delivery Rate (PDR) vs Number of Nodes. 


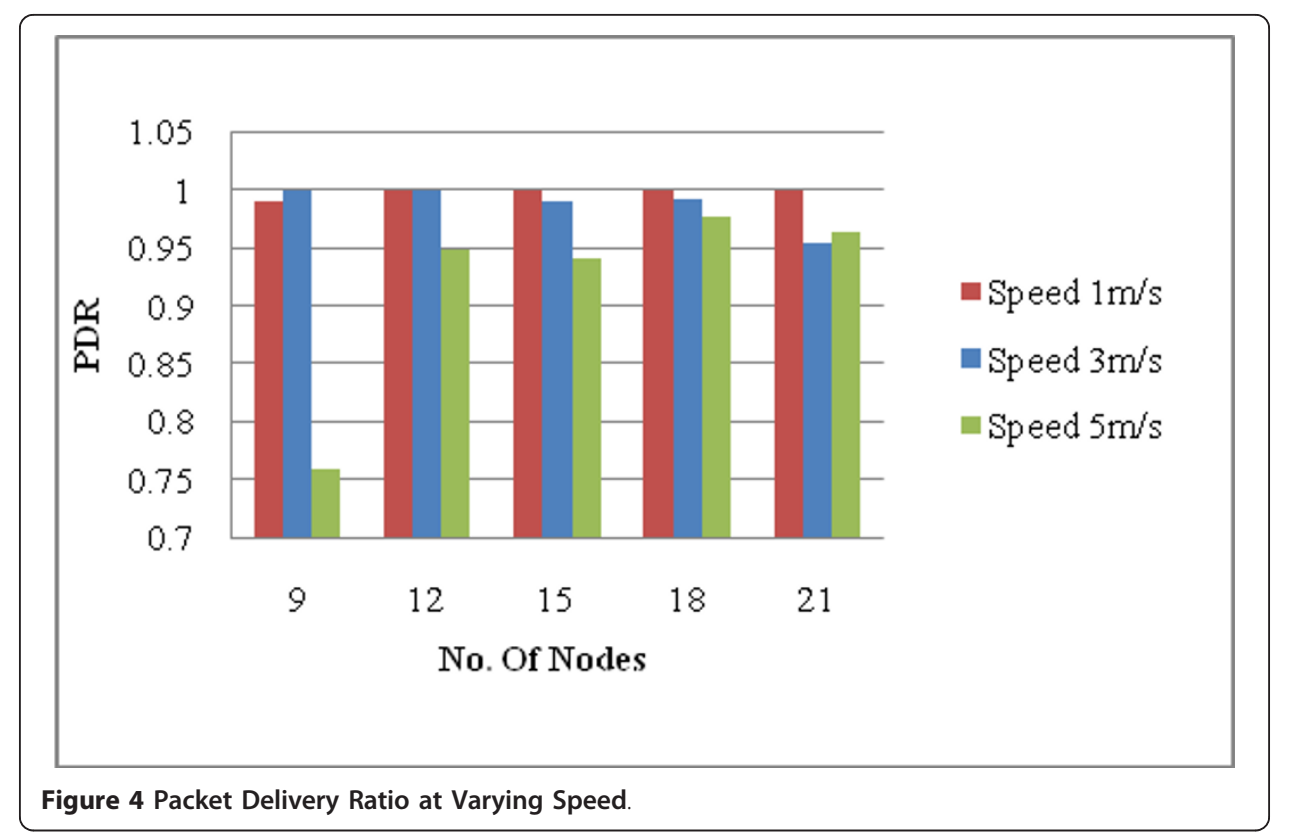

\section{Packet Delivery Ratio in Static and Mobile Scenario}

Figure 3 shows the comparison between the static and mobile environment in terms of the Packet Delivery Ratio (PDR) versus the number of nodes. As shown in Figure 1, in static scenario the packet delivery ratio is between $98 \%$ to $100 \%$, hence guarantees near to $100 \%$ delivery of packets to the geocast region. In the mobile scenario, the lowest packet delivery ratio is $95 \%$. The mobile scenario presents various packet delivery ratios as the nodes are moving randomly in the simulated environment. Due to the random movements, the nodes are moving in and out of the geocast region as well as on the selected route to the geocast region from the source node and this affects the packet delivery ratio. From Figure 3, it can be concluded that the proposed algorithm succeeds in achieving the packet delivery ratio on an average between $95 \%-100 \%$ in the mobile scenario.

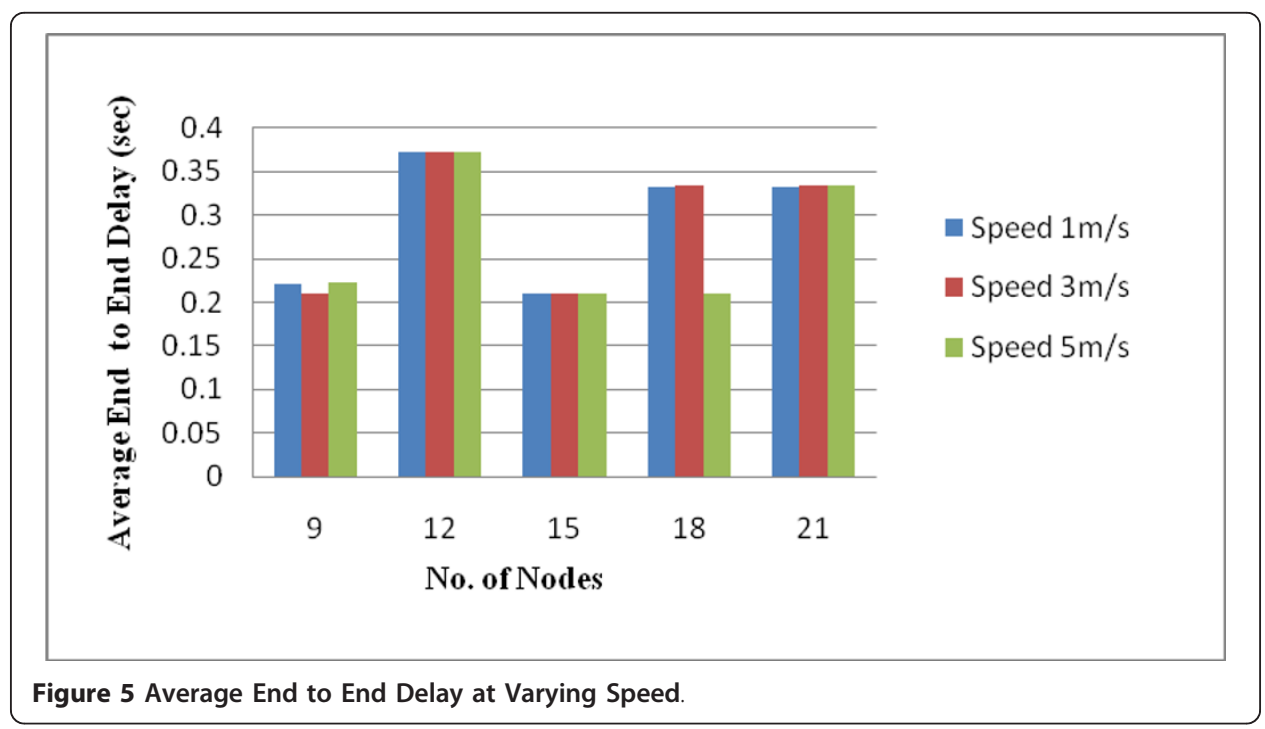




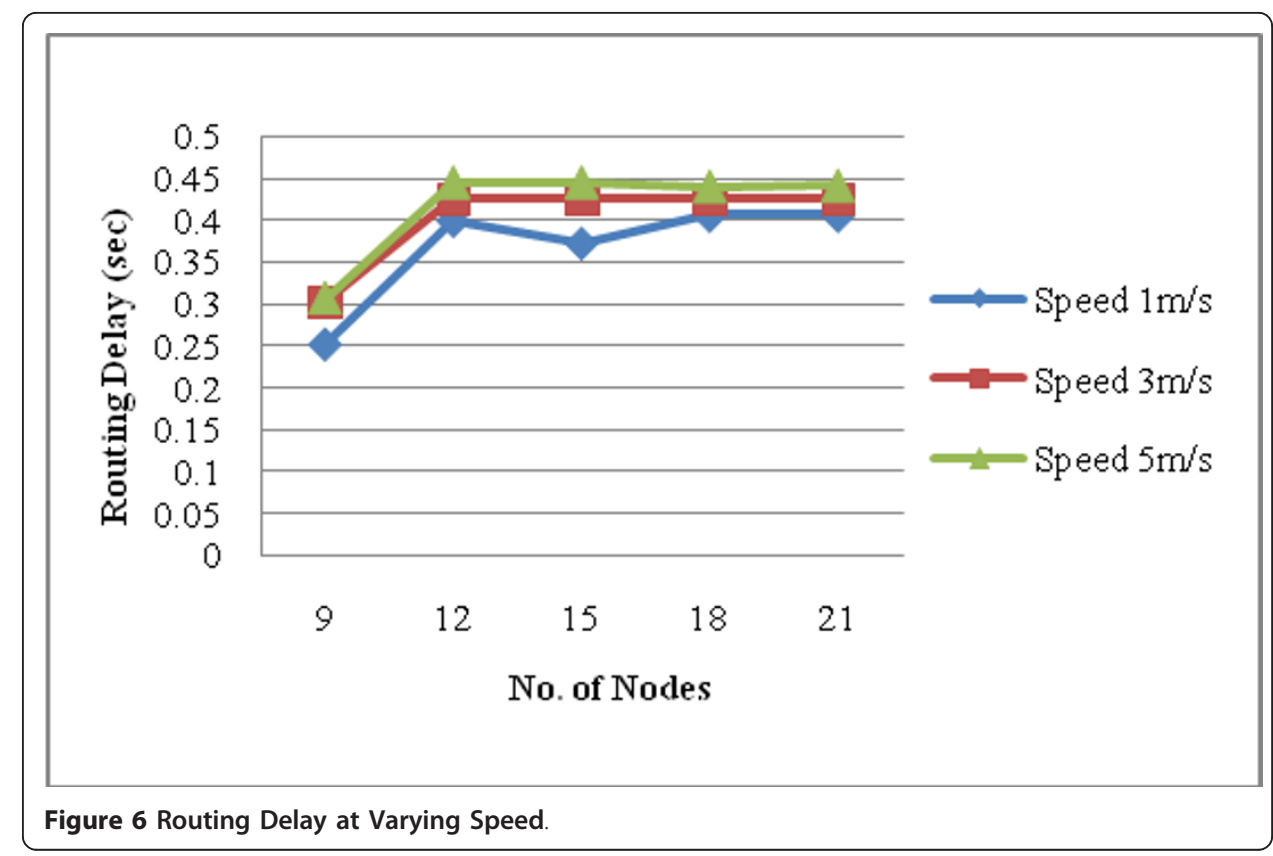

Packet Delivery Ratio (PDR) in Mobile Scenario with Varying Speed

In Figure 4, the packet delivery ratio graph is represented at various speeds. When the nodes are moving at speed of $5 \mathrm{~m} / \mathrm{s}$, the average packet delivery ratio is between $75 \%$ 98\%. As shown in the Figure when the number of nodes is 18 at speed of $5 \mathrm{~m} / \mathrm{s}$, the PDR is $98 \%$. In the figure when the number of nodes is 9, the PDR drops to the lowest value of $76 \%$, as there was a link break that occurred between a pair of nodes. This leads to the re-establishment of the route which ultimately could not be established. This was due to the fact that there were less number of nodes in the region and the nodes were highly mobile. At the speed of $3 \mathrm{~m} / \mathrm{s}$, the packet delivery ratio is between $95 \%-100 \%$. As shown in Figure 4, when the scenario is at high mobility with a speed of

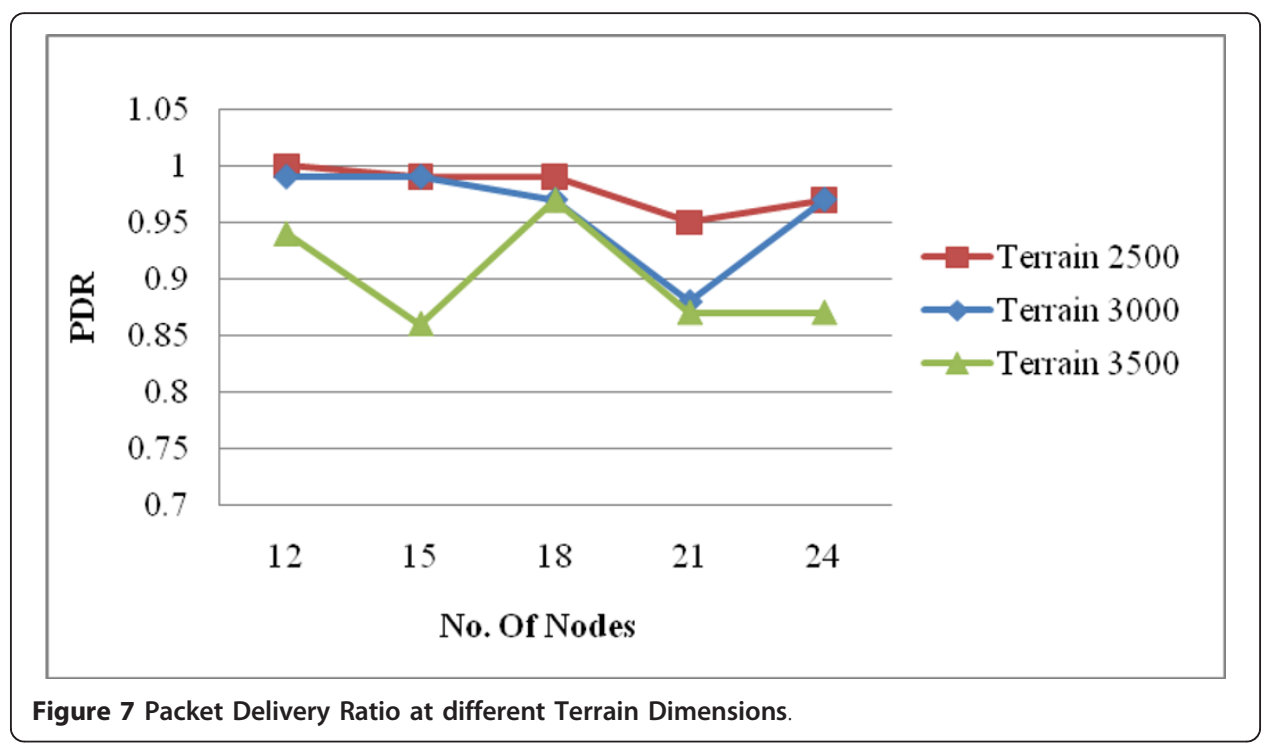




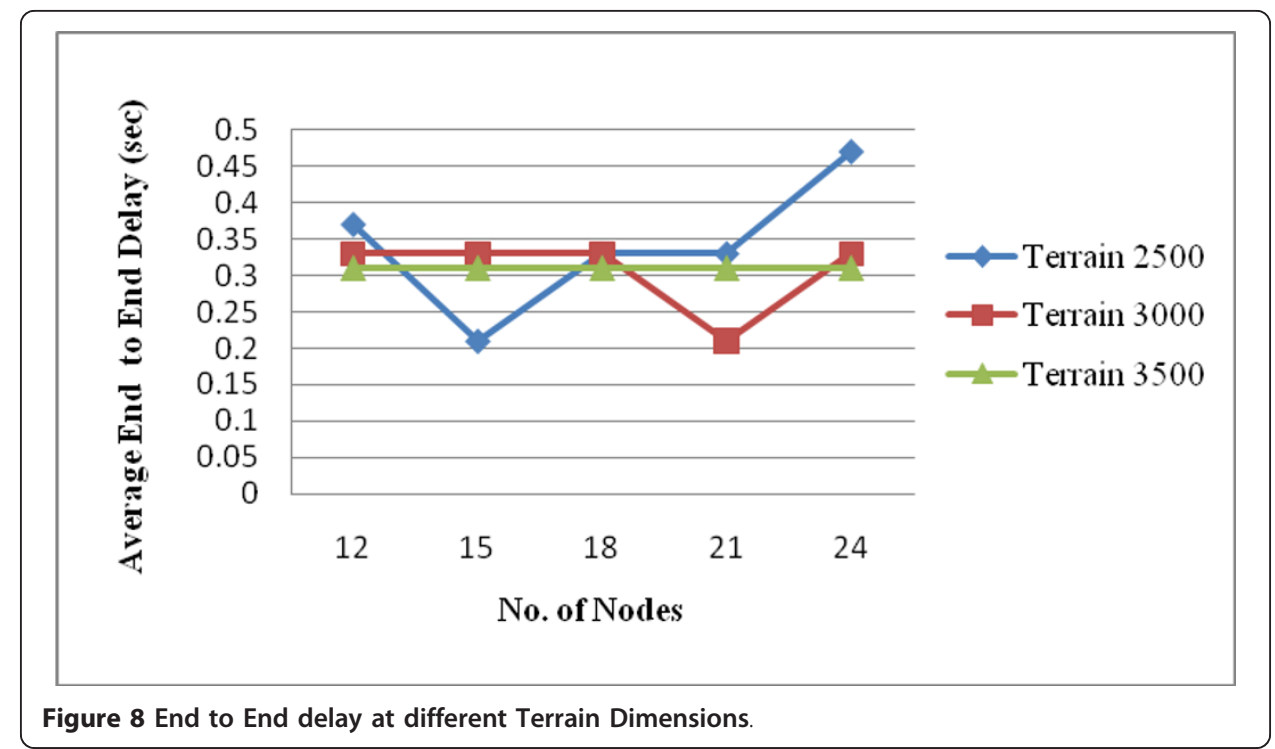

$5 \mathrm{~m} / \mathrm{s}$ and $3 \mathrm{~m} / \mathrm{s}$, the packet delivery ratio falls to $96 \%$. At the speed of $1 \mathrm{~m} / \mathrm{s}$ (which is the usual speed of nodes in underwater current), the packet delivery ratio is almost $100 \%$ in all cases. From the graph plotted in Figure 4, it can be concluded that in any type of mobile scenario, the average packet delivery ratio is between $95 \%-100 \%$. And in sparse network condition, it is approximate to $75 \%$.

\section{Average End to End Delay in Mobile Scenario with Varying Speed}

In Figure 5, the average end to end delay graph is represented at various speeds. When the nodes are moving at the speed of $5 \mathrm{~m} / \mathrm{s}$, the average boundary routing delay is between $0.2 \mathrm{~s}-0.38 \mathrm{~s}$. In the sparse network condition, when the number of nodes is low, at various speeds, the delay is $0.2 \mathrm{~s}$. In one scenario, when the number of nodes is 12 at various speed the delay reaches $0.38 \mathrm{~s}$. This is due to larger broadcasting and data delivery jitter (variation in delay) introduced to avoid collisions in the network. In

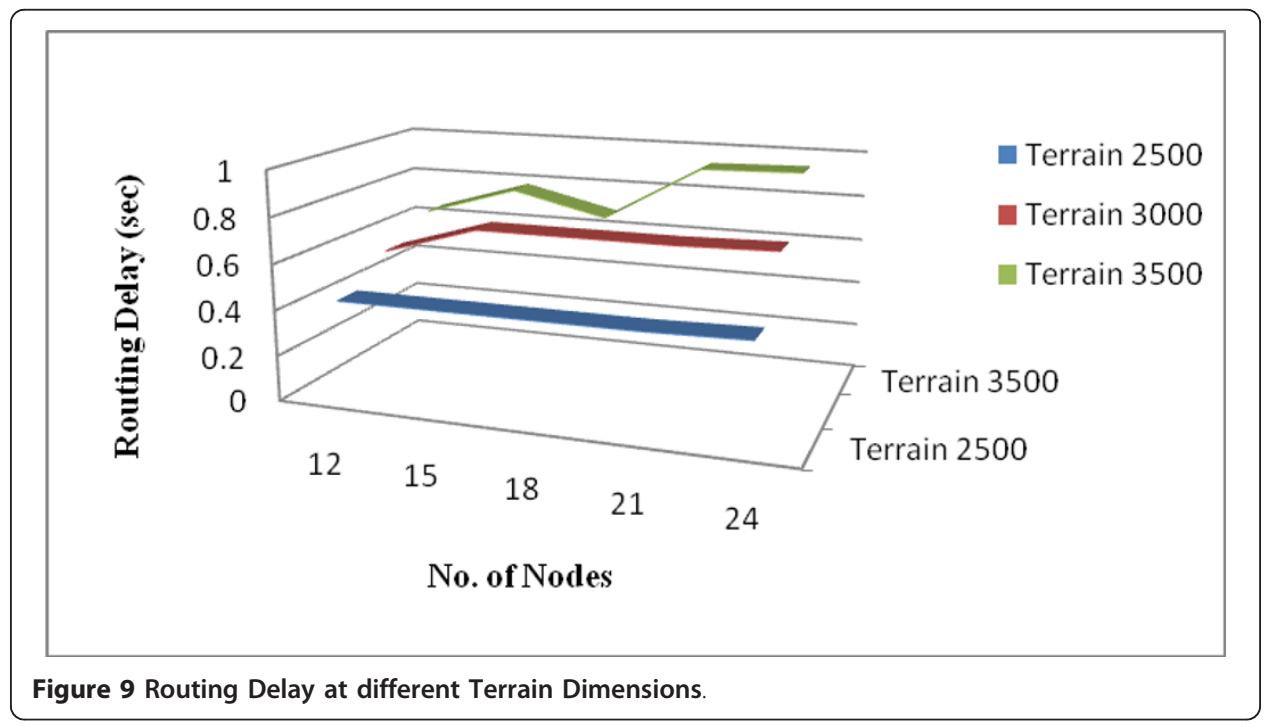




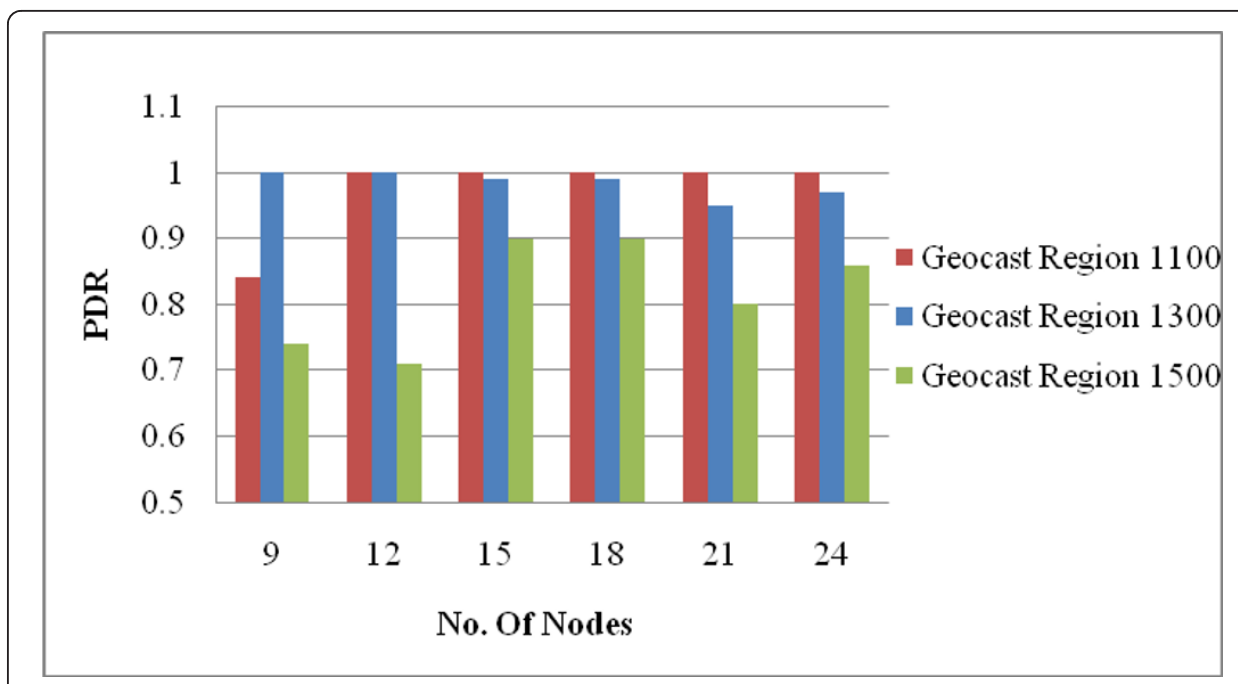

Figure 10 Packet Delivery Ratio at different Geocast Region Dimensions.

all other conditions it works normally. It can be concluded that as the number of nodes are increasing in the region, delay is increasing as well. This is because of the time needed to get the data to the unreached nodes of the geocast region.

\section{Routing Delay in Mobile Scenario with Varying Speed}

In Figure 6, the routing delay graph is represented at various speeds. From the graph it can be inferred that as the nodes speed increases, time taken to reach the geocast region increases as well. This is because in order to avoid collisions in the network, jitter needs to be adjusted appropriately. Increasing the various parameters values affects the delay to root node of the geocast region from the source node. Minimum delay from source node to root node is $0.26 \mathrm{sec}$ at a speed of $1 \mathrm{~m} / \mathrm{s}$ and the maximum delay is $0.45 \mathrm{sec}$ at a speed of $5 \mathrm{~m} / \mathrm{s}$.

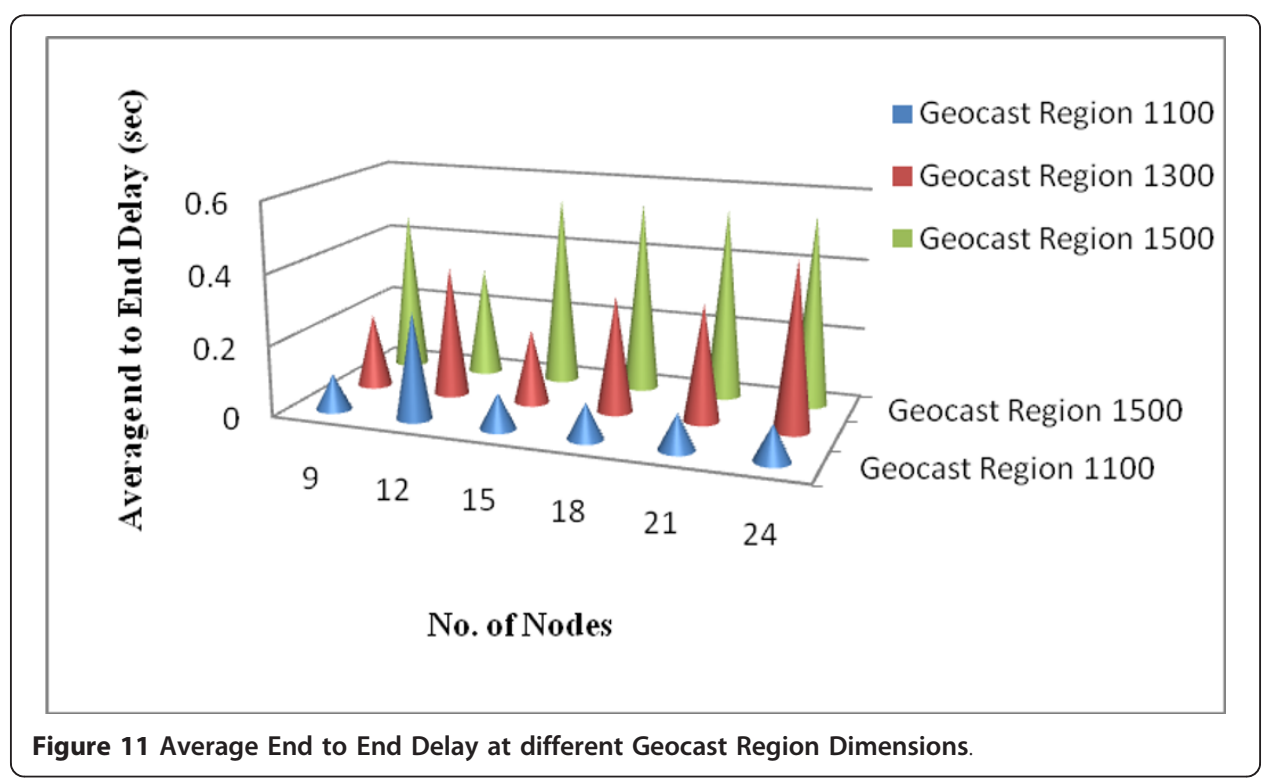




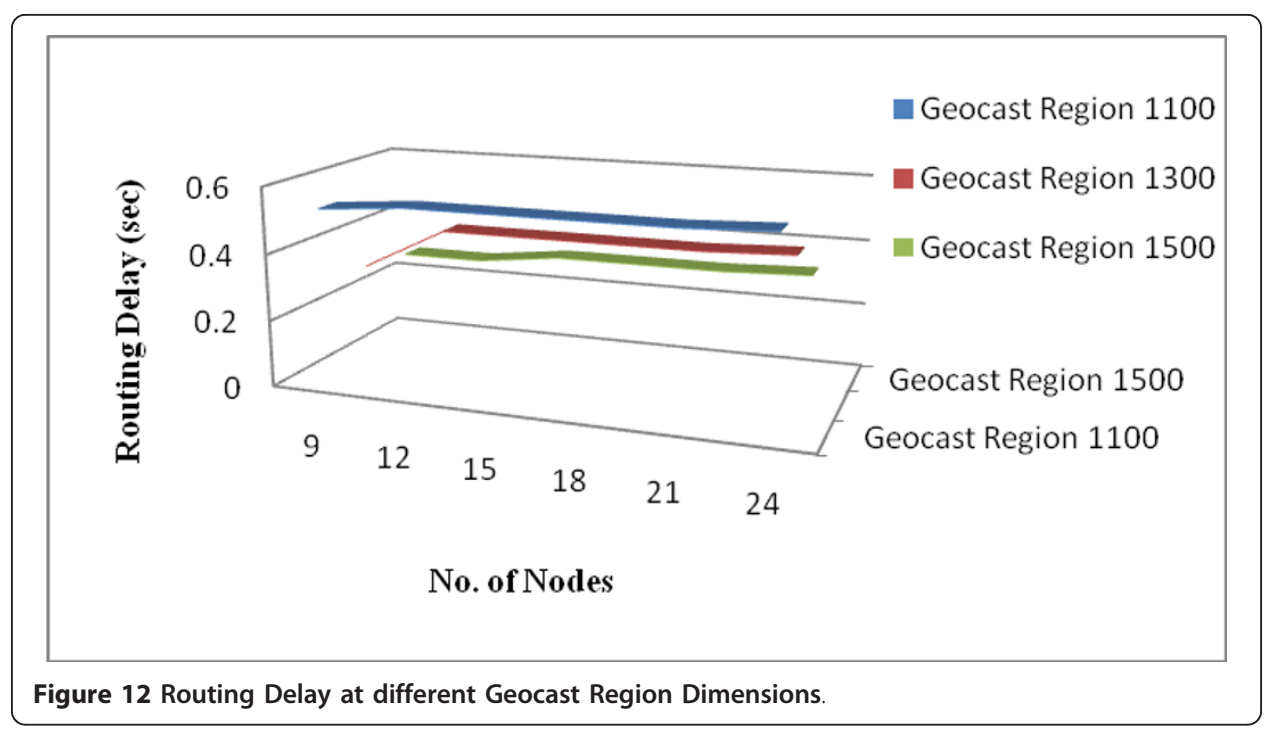

Packet Delivery Ratio in Mobile Scenario with different Terrain dimensions

The packet delivery ratio graph is represented in Figure 7 at different terrain dimensions. The terrain dimensions specified in the graph as "Terrain 2500" implies a terrain dimension of $2500 \times 2500$ square meters. The same is to be considered for all other cases. At the terrain dimension of $3500 \times 3500$ square meters, the average packet delivery ratio is between $86 \%-97 \%$; in the scenario where the number of the nodes is 15 , the PDR falls to $86 \%$ due to the sparse network condition. At the smaller terrain dimension of $2500 \times$ 2500 square meters, the average packet delivery ratio is between $95 \%-100 \%$. From the graph it can be concluded that in different terrain dimensions $2500 \times 2500,3000 \times 3000$ and $3500 \times 3500$ the average packet delivery ratio is between $85 \%-100 \%$.

\section{Average End to End Delay in Mobile Scenario with different Terrain dimensions}

The average end to end delay graph is represented in Figure 8 at different terrain dimensions. End to End delay graph represents the delay involved in the data transfer within the geocast region, from the root node to the last leaf node of the multicast tree. For terrain dimensions of $3500 \times 3500$ and $3000 \times 3000$ square meters, this delay is almost same that is $0.3 \mathrm{sec}$. When the terrain is of smaller dimension, $2500 \times 2500$ square meters, more nodes come in the vicinity of the targeted geocast region and hence the average end to end delay is affected, as shown in the graph. This value in this case varies from $0.2 \mathrm{sec}$ to $0.47 \mathrm{sec}$.

\section{Routing Delay in Mobile Scenario with different Terrain dimensions}

The routing delay graph is represented in Figure 9 at different terrain dimensions. It is clear from the figure that as the terrain dimension increases, the average routing delay also increases. This is so because when the terrain size increases, the routing path length increases too and hence it affects the routing delay from the source node to the root node of the geocast region. The routing delay is directly proportional to the terrain size. For the terrain of size $2500 \times 2500$ square meters, the routing delay is lower $0.43 \mathrm{sec}$ only, while at a larger terrain of size $3500 \times 3500$ square meters, the routing delay increases to $0.94 \mathrm{sec}$. 
Packet Delivery Ratio(PDR)in Mobile Scenario with different Geocast Region dimensions In Figure 10, the packet delivery ratio graph is represented at the different geocast region dimensions. The geocast region dimensions specified in the graph as "Geocast Region 1100" implies a geocast region of $1100 \times 1100$ square meters. The same is to be considered for all the cases. When the geocast region is small, in such a scenario less number of nodes exists in the geocast region. For example from the figure we can infer that in case of $1100 \times 1100$ square meters dimension of the geocast region, PDR is $100 \%$ in almost all the cases. The red colored bar in the graph is depicting the geocast region of dimension $1100 \times 1100$ square meters. As the geocast region size increases the packet delivery ratio achieved reaches from $71 \%$ to $100 \%$. In all other scenarios with geocast region dimensions being varied at $1100 \times 1100,1300 \times 1300$ and $1500 \times 1500$, the average packet delivery ratio is above $85 \%$.

\section{Average End to End Delay in Mobile Scenario with different Geocast Region dimensions}

The average end to end delay is represented in Figure 11 at the different geocast region dimensions. It can be observed from the graph that for larger geocast region such as $1500 \times 1500$ square meters, the average end to end delay is $0.54 \mathrm{sec}$, while for geocast region with dimension $1300 \times 1300$ square meters, the average end to end delay is $0.3 \mathrm{sec}$. For the smaller geocast region dimension $1100 \times 1100$ square meters, the average delay is $0.1 \mathrm{sec}$. Finally, from the graph it can be concluded that as the geocast region dimension is increasing the average end to end delay is also increasing as more number of nodes comes in the vicinity of geocast region dimension and hence leading to a larger delay for the data to reach the farthest node from the root node. The End to End delay is directly proportional to the size of the geocast region.

\section{Routing Delay in Mobile Scenario with different Geocast Region dimensions}

The routing delay graph is represented in Figure 12 at different geocast region dimensions. It is clear from the Figure that as the geocast region dimension increases the average routing delay decreases. This is so because, when the geocast region size increases, the routing path length decreases too and hence affects the routing delay from the source node to the root node of the geocast region. The routing delay is inversely proportional to the geocast region size. For the geocast region of size $1100 \times$ 1100 square meters, the routing delay is the highest; that is $0.55 \mathrm{sec}$, while at larger geocast region of size $1500 \times 1500$ square meters, the routing delay decreases to $0.3 \mathrm{sec}$.

\section{Conclusion \& Future work}

This paper has presented an underwater acoustic communication based simulator AQUA-GLOMO. Simulation tools that are available for acoustic networks beneath the big water bodies are very costly and difficult to work with. Hence for our work, we have taken an available open source simulation tool, the GLOMOSIM, as our base tool and implemented the acoustic channel model within it for Under Water Senor Networks (UWSN). We have simulated the underwater Geocasting model RMTG using this simulator under different scenarios. The results achieved in terms of packet delivery ratio, end to end delay and routing delay, have proved the effectiveness of the AQUA-GLOMO simulator. The observations are found to be very encouraging. This 
work is a promising simulator for researchers working in the area of underwater acoustic communications.

We have upgraded the Glomosim simulator's physical and network layer models to make it feasible for underwater communication. For underwater acoustic communication, the main focusing area is communication at the physical layer and the routing between the nodes at the network layer. These two areas present major challenges for researchers to work on.

In the future, we will be enhancing this AQUA-GLOMO simulator with more limiting parameters of acoustic communication and include other useful features that can aid in the testing, verification and validation processes. More underwater network layer routing protocols can also be developed easily on this simulator. We will also be focusing towards implementing the MAC layer protocol for underwater communication in this simulator.

\section{Author details}

${ }^{1}$ Division of InformationTechnology, Netaji Subhas Institute of Technology, University of Delhi, New Delhi ${ }^{2}$ Department of Computer Science \& Software Engineering, Monmouth University, NJ, USA ${ }^{3}$ Division of Computer Engineering, Netaji Subhas Institute of Technology, University of Delhi, New Delhi

\section{Authors' contributions}

SKD initiated, drafted the underwater studies and finalized the manuscript. MG designed the study, performed the statistical studies and drafted the manuscript. MSO has been involved in revising the manuscript and actively coordinating the discussions and the work. All authors read and approved the final manuscript.

\section{Competing interests}

The authors declare that they have no competing interests.

Received: 2 November 2011 Accepted: 7 March 2012 Published: 7 March 2012

\section{References}

1. Dhurandher KSanjay, Obaidat SMohammad, Gupta Megha (2011) "Providing Reliable and Link Stability Based Geocasting Model in Underwater Environment". International Journal of Communication Systems Wiley (in press). DOI: $10.1002 /$ dac

2. Samad ASumi, Shenoy SK, Santhosh Kumar G, Pillai PRS (2011) "A Survey of Modeling and Simulation Tools for Underwater Acoustic Sensor Networks". Int J Research and Reviews in Computer Science (IJRRCS) 40-47

3. Sehgal Anuj, Tumar lyad, Schönwälder Jürgen "AquaTools: An Underwater Acoustic Networking Simulation Toolkit". Proceedings of IEEE Oceans Conference, Sydney, May 2010

4. Llor Jesús, Torres Esteban, Garrido Pablo, Malumbres PManuel (2009) "Analyzing the Behavior of Acoustic Link Models in Underwater Wireless Sensor Networks". ACM' PM2HW2N'09. Tenerife, Canary Islands, Spain

5. Takai M, Bajaj L, Ahuja R, Bargrodia R, Gerla M (1999) "GloMoSim: A scalable network simulation environment". Technical Report 990027. Department of Computer Science, University of California, Los Angeles, USA

6. Trujillo Ra'ul Palacios (2010) "Interference Cancellation and Network Coding for Underwater Communication Systems". Wireless Communication project. AALBORG University

7. Liu Lanbo, Zhou Shengli, Cui Jun-Hong (2008) "Prospects and Problems of Wireless Communication for Underwater Sensor Networks". Wiley WCMC Special Issue on Underwater Sensor Networks 8(8):877-994

8. Berkhovskikh L, Lysanov Y (1982) "Fundamentals of Ocean Acoustics". Springer

9. Stojanovic M (2006) "On the relationship between capacity and distance in an underwater acoustic communication channel". Proceedings of the 1st ACM international workshop on Underwater networks. WUWNet ?'?06

10. Zorzi Michele, Casari Paolo, Baldo Nicola, Harris FAlbert III (2008) "Energy-Efficient Routing Schemes for Underwater Acoustic Networks". IEEE Journal on Selected Areas in Communications 26(9):1754-1766

11. Urick R (1983) "Principles of Underwater Sound". McGraw-Hill

12. Sundani Harsh, Li Haoyue, Devabhaktuni KVijay, Alam Mansoor, Bhattacharya Prabir (2011) "Wireless Sensor Network Simulators A Survey and Comparisons". International Journal of Computer Networks (IJCN) 2(5):249-265

doi:10.1186/2192-1962-2-3

Cite this article as: Dhurandher et al:: An acoustic communication based AQUA-GLOMO simulator for underwater networks. Human-centric Computing and Information Sciences 2012 2:3. 